\title{
Bibliographie de l'histoire de l'Amérique française
}

\section{Robert Gagnon, Marie-Ève Blache-Gagné, Élise Detellier, Yannick Gendron, Marie-Christine Giroux, Suzanne Ledoux et Sonya Roy}

Volume 63, numéro 1, été 2009

URI : https://id.erudit.org/iderudit/039901ar

DOI : https://doi.org/10.7202/039901ar

Aller au sommaire du numéro

Éditeur(s)

Institut d'histoire de l'Amérique française

ISSN

0035-2357 (imprimé)

1492-1383 (numérique)

Découvrir la revue

Citer ce document

Gagnon, R., Blache-Gagné, M.-È., Detellier, É., Gendron, Y., Giroux, M.-C., Ledoux, S. \& Roy, S. (2009). Bibliographie de l'histoire de l'Amérique française. Revue d'histoire de l'Amérique française, 63(1), 177-185.

https://doi.org/10.7202/039901ar d'utilisation que vous pouvez consulter en ligne. 


\title{
B I B L I O G R A P H I E \\ DE L' H ISTOIRE DE L'A MÉ R I Q U E FRAN ÇA ISE
}

\author{
ROBERT GAGNON \\ Directeur \\ MARIE-ÈVE BLACHE-GAGNÉ \\ Université du Québec à Montréal \\ ÉLISE DETELLIER \\ Université de Montréal \\ YANNICK GENDRON \\ Collège Laflèche \\ MARIE-CHRISTINE GIROUX \\ Université du Québec à Montréal \\ SUZANNE LEDOUX \\ Bibliothèque nationale \\ SONYA ROY \\ Université McGill
}

Grille de classement

AVANT I600

XVII $^{\mathrm{e}}$ ET XVIII ${ }^{\mathrm{e}}$ SIÈCLES

$\mathrm{XIX}^{\mathrm{e}}$ SIÈCLE

I900-I945

DEPUIS I945

AUTRES

INSTRUMENTS DE TRAVAIL
La grille de classement est chronologique. Les travaux qui couvrent deux ou trois périodes sont classés dans celle à laquelle ils appartiennent principalement ou dans la première. $S$ 'ils touchent à plus de trois périodes, on les trouve dans la section "Autres». 


\section{AVANT 1600}

\section{XVIIe ET XVIIIe SIÈCLES}

BLAIS, Christian, «La représentation en Nouvelle-France», Bulletin d'histoire politique, 18,1 (automne 2009): 51-76.

BLOUIN, Pierre, "Champlain et le Saint-Laurent: un fleuve et un pays », L'Action nationale, 49,5-6 (avril 2009): 21-38.

BOURQUE, Todd J., «L'Hôpital des pauvres to Hospital de los Pobres: New Orleans' Charity Hospital in Spanish Colonial Louisiana », Louisiana History, 50,1 (hiver 2009): 57-70.

CHARLEBOIS, Paul-Yvon, «Les derniers militaires français aux Illinois après la guerre de Sept Ans (1761-1770)", Mémoires de la Société généalogique canadienne-française, 60,3 (automne 2009): 177-188.

CHARTRAND, René, «La gouvernance militaire en Nouvelle-France», Bulletin d'histoire politique, 18,1 (automne 2009): 125-136.

CHEVRIER, Marc, "La République néo-française», Bulletin d'histoire politique, 17,3 (printemps-été 2009): 29-58.

DELÂGE, Denys, "Aux origines de Québec: les malentendus fondateurs », Études canadiennes/Canadian Studies, 66 (2009): 27-45.

DELÂGE, Denys, «Modèles coloniaux, métaphores familiales et logiques d'empire en Amérique du Nord aux XVII ${ }^{\mathrm{e}}$ et XVIII ${ }^{\mathrm{e}}$ siècles", Bulletin d'histoire politique, 18,1 (automne 2009): 103-124.

DESCHÊNES, Gaston, L'année des Anglais : la Côte-du-Sud à l'heure de la conquête (Québec, Septentrion, 2009), 158 p. Nouvelle édition revue et augmentée.

DIN, Gilbert C., «Empires Too Far: The Demographic Limitations of Three Imperial Powers in the Eighteenth-Century Mississippi Valley", Louisiana History, 50,3 (été 2009): 261-292.

GARNEAU, Jean-Philippe, «Rendre justice en Nouvelle-France: les voies et les limites de l'obéissance», Bulletin d'histoire politique, 18,1 (automne 2009): 87-102.

GREER, Allan, La Nouvelle-France et le monde (Montréal, Boréal, 2009), 310 p.

GRENIER, Benoît, «Pouvoir et contre-pouvoir dans le monde rural laurentien aux XviII ${ }^{\mathrm{e}}$ et $\mathrm{xIx}^{\mathrm{e}}$ siècles: sonder les limites de l'arbitraire seigneurial », Bulletin d'histoire politique, 18,1 (automne 2009): 143-163.

HAMEL, Nathalie, «Un papier peint inspiré de l'Orient dans une ville coloniale d'Amérique : présence de la chinoiserie dans la maison Estèbe à Québec au milieu du xviII ${ }^{\mathrm{e}}$ siècle», Revue de culture matérielle/Material Cutlure Review, 68 (automne 2008): 19-33.

MIQUELON, Dave B., "After Ryswick: The Five Nations Iroquois in French Diplomacy», Native Studies Review, 18,1 (2009): 5- 24.

MONIÈRE, Denis et Serge BERNIER, dir., et al., La gouvernance en Nouvelle-France (Montréal, VLB, 2009), 321 p. 
MORGAN, M. J., «Indians on Trial: Crime and Punishment in French Louisiana on the Eve of the Seven Years' War", Louisiana History, 50,3 (été 2009): 293-320.

MORIN, Maxime, «L'abbé Pierre Maillard: une figure missionnaire emblématique du XVIII ${ }^{\mathrm{e}}$ siècle acadien", SCHEC, Études d'histoire religieuse, 75 (2009): 39-54.

NERICH, Laurent, La petite guerre et la chute de la Nouvelle-France (Outremont, Athéna éditions, coll. «Histoire militaire», 2009), 243 p.

PUSCH, Donald E., «Kerlérec's Cipher: The Code Book of Louisiana’ Last French Governor ", Louisiana History, 49,4, (automne 2008): 463-480.

ROBICHAUD, Léon, «Les réseaux d’influence à Montréal au XviI siècle», Bulletin d'histoire politique, 18,1 (automne 2009): 77-86.

SAYRE, Gordon M., «Natchez Ethnohistory Revisited: New Manuscript Sources from Le Page du Pratz and Dumont de Montigny ", Louisiana History, 50,4 (automne 2009): 407-436.

STACEY, C. P., Québec, 1759: le siège et la bataille (Québec, Les Presses de l’Université Laval, 2009), xxix-329 p.

STANDEN, Dale, «Canoes and Canots in New France: Small Boats, Material History and Popular Imagination", Revue de culture matérielle/Material Cutlure Review, 68 (automne 2008): 34-47.

TREMBLAY, Yves, Plaines d'Abraham. Essai sur l'égo-mémoire des Québécois (Outremont, Athéna éditions, coll. «histoire militaire», 2009), 252 p.

VILLERBU, Tangi, «Pouvoir, religion et société en des temps indécis: Vincennes, 17631795 ", Revue d'histoire de l'Amérique française, 62,2 (automne 2008) : 185-214.

WILHELMY, Jean-Pierre, Les mercenaires allemands au Québec, 1776-1783 (Québec, Septentrion, 2009), 276 p. Nouvelle édition.

\section{SIÈCLE}

BÉDARD, Éric, Les réformistes: une génération canadienne-française au milieu du XIX ${ }^{\mathrm{e}}$ siècle ([Montréal], Boréal, 2009), 415 p.

CLICHE, Marie-Aimée, «Les limbes. Opinions théologiques et croyances populaires au Québec du $\mathrm{xvII}^{\mathrm{e}}$ au $\mathrm{xx}^{\mathrm{e}}$ siècle», Revue d'histoire de l'Amérique française, 62,3-4 (hiverprintemps 2009): 351-376.

DEFERT, Jean-Jacques et Claude COUTURE, Récits $d u$ XIX ${ }^{\mathrm{e}}$ siècle : structure et contenu $d u$ discours historiographique au Canada au XIX ${ }^{\mathrm{e}}$ siècle: Garneau, Kingsford, Rameau de Saint Père, Smith (Québec, Les Presses de l’Université Laval, coll. «Prisme», 2009), ix-147 p.

DESSUREAULT, Christian, «L'émeute de Lachine en 1812: la coordination d’une contestation populaire ", Revue d'histoire de l'Amérique française, 62,2 (automne 2008): 215-252.

DUGAS, Kathy, «An Immigrant's Journey to Wealth and Power: The Story of FrançoisXavier Martin», Louisiana History, 50,3 (été 2009): 321-340.

GAUVREAU, Michael, «Forging a New Space for Lay Male Piety: St. Vincent de Paul Societies in Urban Quebec and Ontario, 1846-1890", Histoire sociale/Social History, 47,83 (mai 2009): 35-68. 
HARVEY, Louis-Georges, "Les Patriotes, le républicanisme et la constitution québécoise », Bulletin d'histoire politique, 17,3 (printemps-été 2009): 59-77.

INGRAM, Darcy, “"Au temps et dans les quantités qui lui plaisent” : Poachers, Outlaws, and Rural Banditry in Quebec», Histoire sociale/Social History, 47,83 (mai 2009): 1-36.

KAUPP, Dorothée, “"Nos premiers missionnaires": l'histoire des récollets dans les ouvrages franciscains au Canada, $\mathrm{XIX}^{\mathrm{e}}-\mathrm{xx}^{\mathrm{e}}$ siècles", Études d'histoire religieuse, 75 (2009): 25-38.

POULTER, Gillian, Becoming Native in a Foreign Land: Sport, Visual Culture and Identity in Montreal, 1840-85 (Vancouver, UBC Press, 2009), xi-374 p.

SAINT-PIERRE, Jocelyn, «La présence de la Nouvelle-France dans l'hôtel du Parlement», Bulletin d'histoire politique, 18,1 (automne 2009): 9-18.

TÉSIO, Stéphanie, Histoire de la pharmacie en France et en Nouvelle-France au XVIII siècle (Québec, Les Presses de l’Université Laval, 2009), xxx-331 p.

\section{$1900-1945$}

COURTOIS, Charles-Philippe, «Le républicanisme au Québec au début du xx ${ }^{\mathrm{e}}$ siècle : le cas de figure de Wilfrid Gascon, Olivar Asselin et Ève Circé-Côté», Bulletin d'histoire politique, 17,3 (printemps-été 2009): 93- 120.

DETELLIER, Élise, “"Bonifier le capital humain”. Le genre dans le discours médical et religieux sur les sports au Québec, 1920-1950", Revue d'histoire de l'Amérique française, 62,3-4 (hiver-printemps 2009): 473-500.

FAUGIER, Étienne, «Automobile, transports urbains et mutations : l'automobilisation urbaine de Québec, 1919-1939", Revue d'histoire urbaine/Urban History Review, 37,1 (automne 2009): 26-38.

GÉLINAS, Claude, «Les Autochtones et le partenariat économique au Québec, 18671960 », Recherches amérindiennes au Québec, 38,1 (2008): 29-40.

GINGRAS, Yves, «Duns Scot vs Thomas d'Aquin. Le moment québécois d'un conflit multi-séculaire", Revue d'histoire de l'Amérique française, 62,3-4 (hiver-printemps 2009): 377-406.

KESHEN, Jeffrey, Saints, Salauds et Soldats. Le Canada et la Deuxième Guerre mondiale (Outremont, Athéna éditions, coll. «Histoire militaire», 2009), 428 p.

LACOMBE, Sylvie, «Fils légitimes de l'imaginaire national: les Canadiens français selon The Beaver-Canada First, organe des Native Sons of Canada, 1928-1929», Mens, Revue d'histoire intellectuelle de l'Amérique française, 9,2 (printemps 2009): 207-241.

LAMONDE, Yvan, "La rage de Vivre et les "cahiers noirs" (1934-1935)», Mens, Revue d'histoire intellectuelle de l'Amérique française, 9,2 (printemps 2009): 187-206.

MARQUIS, Dominique, "La Revue dominicaine, 1915-1961. Un regard catholique sur une société en mutation", Revue d'histoire de l'Amérique française, 62,3-4 (hiver-printemps 2009): 407-428. 
MASSICOTTE, Julien, «Portrait d'un “fondateur dans l'âme”: Clément Cormier, pionnier des sciences sociales en Acadie du Nouveau-Brunswick", Acadiensis, 38,1 (hiver/ printemps 2009): 3-32.

PFEIFER, Michael J., "The Origins of Postbellum Lynching: Collective Violence in Reconstruction Louisiana", Louisiana History, 50,2 (printemps 2009): 189-201.

POUTANEN, Mary Anne, Sherry OLSON, Raphaël FISCHLER et Kevin SCHWARTZMAN, "Tuberculosis in town: Mobility of Patients in Montreal, 19251950 », Histoire sociale/Social History, 47,83 (mai 2009): 69-108.

ST-ONGE, Nicole, «Memories of Metis Women of Saint-Eustache, Manitoba - 19101980 », Native Studies Review, 17,2 (2008): 45-68.

VINCENT, Charles, "“Of Such Historical Importance...”: The African American Experience in Louisiana », Louisiana History, 50,2 (printemps 2009): 133-158.

\section{DEPUIS 1945}

ABICH, Kristina et Daniel BÉLAND, «Policy Change and the Politics of Ideas: The Emergence of the Canada/Quebec Pensions Plans», Revue canadienne de sociologie/ Canadian Review of Sociology, 46,3 (août 2009): 253-271.

BALTHAZAR, Louis, «La nation québécoise en évolution: la dynamique de la multiethnicité", Études canadiennes/Canadian Studies, 66 (2009): 47-62.

BHERER, Claude, Bernard BRAIS et Hélène VÉZINA, «Impact des récentes transformations démographiques liées à l'urbanisation sur le bassin génétique de la région de Lanaudière», Cahiers québécois de démographie, 37,2 (automne 2008): 211- 236.

COURTOIS, Charles-Philippe, «Pour un rééquilibrage de l'historiographie et de la représentation de la Nouvelle-France dans l'enseignement de l'histoire », Bulletin d'histoire politique, 18,1 (automne 2009): 19-36.

DASSYLVA, Martial, “"Le meilleur système d'éducation au monde”. Omer-Jules Desaulniers et l'idéologie scolaire catholique ", Revue d'histoire de l'Amérique française, 62,3-4 (hiver-printemps 2009): 501-532.

DE SURMONT, Jean-Nicolas, «Francophonie et régionalisme: entre une vision universalisante et une version particularisante de la langue française », Bulletin d'histoire politique, 17,3 (printemps-été 2009): 195-218.

DUFOUR, Pascale et Christophe TRAISNEL, "Aux frontières mouvantes des mouvements sociaux, ou quand les partis politiques s'en mêlent. Le cas du souverainisme au Québec», Politiques et Sociétés, 28,1 (2009): 37-63.

FAVREAU, Louis, "Coopération internationale de proximité. Histoire, fondements et enjeux actuels des OCI du Québec», Globe, Revue internationale d'études québécoises, 12,1 (2009): 17-42.

FERRETTI, Lucia, «Reynald Rivard, prêtre psychologue trifluvien, l'essor de l'éducation spécialisée au Québec et la fin des orphelinats ordinaires (1947-1968)", Revue d'histoire de l'Amérique française, 62,3-4 (hiver-printemps 2009): 533-564. 
GAGNON, Alain-G., «Révolutionnaires en quête d'un métarécit: de la société québécoise global à la citoyenneté québécoise», Études canadiennes/Canadian Studies, 66 (2009): 27-45.

GAUVREAU, Michael, «Catholicisme, nationalisme et fédéralisme dans la pensée de Claude Ryan: la contribution de l'Action catholique, 1945-1964», Revue d'histoire de l'Amérique française, 62,3-4 (hiver-printemps 2009) : 429-472.

GÉLINAS, Xavier, «D’une droite à l’autre : le conservatisme intellectuel canadien-français des années 1960 et 2000 », Études canadiennes/Canadian Studies, 66 (2009): 63-74.

GENDRON, Robin S., «"Le prestige du Canada en jeu.” Le père Lévesque et l’Université nationale du Rwanda dans les années 1960 : entre le Canada et le Québec», Globe, Revue internationale d'études québécoises, 12,1 (2009): 95-114.

GROULX, Lionel-Henri, «La restructuration récente des politiques sociales au Canada et au Québec: éléments d'analyse», Le Travail/Labour, 63 (printemps 2009): 9-46.

GUENIN-LELLE, Dianne et Alison HARRIS, "The Role of Music Festivals in the Cultural Renaissance of Southwest Louisiana in the Late Twentieth Century ", Louisiana History, 50,4 (automne 2009): 461-472.

HUDON, Raymond, Rachel MATHIEU et Élisabeth MARTIN, "Pouvoir médical et interventions législatives au Québec, 2001-2008", Recherches sociographiques, 50,2 (maiaoût 2009): 255-282.

LABELLE, Micheline, «Les intellectuels québécois face au multiculturalisme: hétérogénéité des approches et des projets politiques», Études ethniques au Canada / Canadian Ethnic Studies, 40,1 (2008): 33-56.

LAFORTUNE, Gina et Fasal KANOUTÉ, «Vécu identitaire d'élèves de $1^{\text {re }}$ et de $2^{\mathrm{e}}$ génération d'origine haïtienne », Revue de l'Université de Moncton, 38,2 (2007): 33-71.

LAVEAULT, Jonathan, «Le long parcours d'un militant nationaliste: Rosaire Morin (1923-1999)", Bulletin d'histoire politique, 17,3 (printemps-été 2009): 155-170.

LEGRAND, Catherine, «L'axe missionnaire catholique entre le Québec et l’Amérique latine. Une exploration préliminaire», Globe, Revue internationale d'études québécoises, 12,1 (2009): 43-66.

MEREN, David, “"Plus que jamais nécessaires”: Cultural Relations, Nationalism and the State in the Canada-Québec-France Triangle, 1945-1960", Revue de la Société historique du Canada/Journal of the Canadian Historical Association, 19,1 (2008): 279-305.

MESLI, Samy, «Le développement de la "diplomatie éducative” du Québec», Globe, Revue internationale d'études québécoises, 12,1 (2009): 115-132.

OLOFSSON, Ebba, Tara L. HOLTON et Imaapik J. PARTRIDGE, «Negotiating Identities: Inuit Tuberculosis Evacuees in the 1940s-1950s ", Études Inuit/Inuit Studies, 32,2 (2008): 127-149.

PAGÉ, Pierre, «L'image médiatique de l’Église et l'opinion publique au Québec: deux décennies paradoxales de mutations (1968-1984)", Études d'histoire religieuse, 75 (2009): 89-108. 
PAQUETTE, Gilbert, "Camille Laurin : le courage et la détermination », L'Action nationale, 49,4 (avril 2009): 11-25.

POLIQUIN, Daniel, René Lévesque (Montréal, Boréal, 2009), 204 p.

PRUD’HOMME, Julien, «Professionnelles des soins et marchés de la santé. Les trajectoires analogues des physiothérapeutes et des psychologues québécoises, 1950-2010», Revue d'histoire de l'Amérique française, 62,2 (automne 2008) : 253-287.

RICCI, Nino, Pierre Elliott Trudeau (Montréal, Boréal, 2009), 204 p.

ROBERTS, Katherine Ann, «Continental Drift: Negotiating Male Performance in Canadian and American Cinema from the Late Sixties ", American Review of Canadian Studies, 39,1 (mars 2009): 16-28.

ROBINEAU, Anne, «La musique "actuelle” québécoise: une façon distincte de développer une pratique artistique», Études canadiennes/Canadian Studies, 66 (2009): 105 113.

ROY, Martin, «Penser la sortie de la chrétienté au Québec: la contribution de la revue catholique d'opinion Maintenant (1962-1974)", Bulletin d'histoire politique, 17,3 (printemps-été 2009): 171-194.

SERRÉ, Pierre, «L'évolution de la dynamique électorale de 1991 à 2006: le recul du pouvoir francophone», L'Action nationale, 49,5-6 (avril 2009): 59-76.

SIMARD, Martin et Guy MERCIER, "Trente ans d'aménagement institutionnalisé au Québec, une contribution au modèle québécois?", Études canadiennes/Canadian Studies, 66 (2009): 139-151.

SORON, Antony, «Révolution permanente autour du Mont Royal. Lecture analytique des Aurores montréales de Monique Proulx", Études canadiennes/Canadian Studies, 66 (2009): 115-124.

THÉRIAULT, Anne, «Féminisme et nationalisme dans le Québec contemporain, entre modernité et postmodernité», Politique et Sociétés, 28,2 (2009): 53-67.

TREMBLAY, Pierre-André, «Mouvements sociaux, économie sociale, pauvreté : l'expérience québécoise», Études canadiennes/Canadian Studies, 66 (2009): 91-104.

TURGEON, Alexandre, «Entre discussion et polémique: des conceptions de la Cité au sein des pages du Devoir, 1970-1979», Bulletin d'histoire politique, 18,1 (automne 2009): 169-182.

\section{Autres}

AIRD, Robert, «La nation québécoise et l'autodérision », Bulletin d'histoire politique, 17,3 (printemps-été 2009): 219- 232.

ALLARD, Michel et Félix BOUVIER, avec la collaboration de Bernard LEFEBVRE, André Lefebvre, didacticien de l'histoire (Sillery, Septentrion, 2009), $170 \mathrm{p}$.

ANDERSON, Kim, «The Mother Country: Tracing Intersections of Motherhood and the National Story in Recent Canadian Historiography ", Revue d'études sur les femmes / A Women's Studies Journal, 34,1 (2009): 121-131. 
BLANCHETTE, Roger, L'Outaouais (Québec, Les Presses de l’Université Laval, coll. «Les régions du Québec. Histoire en bref ", nº 12, 2009), 180 p.

CARDINAL, Linda, «Le Québec et le monde atlantique», Bulletin d'histoire politique, 17,3 (printemps-été 2009): 17-28.

CHANG, Yuho, La famille et identité dans le roman québécois $d u x x^{\mathrm{e}}$ siècle (Sillery, Septentrion, 2009), $262 \mathrm{p}$.

DUGAS, Jean-Yves, «Histoire des gentilés VI : la gentilité comme moteur de l'identité», L'action nationale, 49,4 (avril 2009): 26-30.

HARVEY, Kathryn, «Location, Location, Location: David Ross McCord and the Makings of Canadian History", Revue de la Société historique du Canada/Journal of the Canadian Historical Association, 19,1 (2008): 57-81.

HÉTU, Luc, Une ville sous tutelle: brève histoire de l'administration de Montréal (Montréal, Liber, 2009), $160 \mathrm{p}$.

LAFOREST, Daniel, "The Blurry Outlines of Historical Urban Space: Quebec City's $400^{\text {th }}$ Anniversary, Its Literary Status and its Suburbs", British Journal of Canadian Studies, 22,2 (2009): 197-213.

LAPERLE, Dominique, “"Revenir sur le chemin parcouru et les jours anciens”. Analyse des textes commémoratifs de quatre Jubiles des Sœurs des Saints Noms de Jésus de Marie (1894, 1944, 1969, 1994)", Études d'histoire religieuse, 75 (2009): 55-68.

LAPERRIÈRE, Guy, Les Cantons-de-l'Est (Québec, Les Presses de l'Université Laval, coll. «Les régions du Québec, Histoire en bref/INRS, n 13, 2009), 197 p.

LÉVESQUE, Benoît, «Les relations internationales de l'économie sociale au Québec sur plus d'un siècle et demi (1840-2008)", Globe, Revue internationale d'études québécoises, 12,1 (2009): 67-94.

LUKIC, Renéo et Serge BERSTEIN, dir., et al., Conflit et coopération dans les relations francoaméricaines: du général de Gaulle à Nicolas Sarkozy (Québec, Les Presses de l'Université Laval, coll. «Politique étrangère et sécurité», 2009), xxvi-366 p.

MORTON, Desmond, Histoire militaire du Canada (Outremont, Athéna éditions, coll. «Histoire militaire», 2009), 380 p. Nouvelle édition.

MOSS, Jane, «Francophone Theater of Western Canada: Dramatic Tales of Disappearing Francophones ", American Review of Canadian Studies, 39,1 (mars 2009): 29-37.

NADEAU, Jean-François, Robert Rumilly, l'homme de Duplessis (Montréal, Lux, coll. «Histoire politique», 2009), $410 \mathrm{p}$.

OUELLET, Marie-Ève, «Structures et pratiques dans l'historiographie de l'État en Nouvelle-France », Bulletin d'histoire politique, 18,1 (automne 2009): 37-50.

PICHETTE, Robert, «Sanctifié dans la captivité; vivifié par la foi : l’Église en Acadie et la commémoration des événements de 1755 », Études d'histoire religieuse, 75 (2009): 109-124.

ROY, Jean et Lucia FERRETTI, dir., Nouvelles pages trifluviennes (Québec, Septentrion, 2009), $339 \mathrm{p}$. 
TRUDEAU, Samuel, «L'expérience républicaine de la guerre, de l'hoplite au désarmement québécois", Bulletin d'histoire politique, 17,3 (printemps-été 2009): 79-92.

TRUDEL, Marcel, Deux siècles d'esclavage au Québec ([Montréal], Bibliothèque québécoise, 2009), 359-[13] p. Nouvelle édition préparée par Micheline D’Allaire.

WADE, Michael G., «Does Lousiana’s Past Have a Future?: The Challenge of the Present», Louisiana History, 50,4 (automne 2009): 389-406.

\section{Insruments de travail}

HUCHON, Mireille, Le français au temps de Jacques Cartier (Rimouski, Tangence, Université du Québec à Rimouski; [Trois-Rivières], Université du Québec à Trois-Rivières, coll. «Confluences», 2009), 186 p. 